\title{
Prediction of Regional Logistics Heat and Coupling Development between Regional Logistics and Economic Systems
}

\author{
Guojun Yin $\mathbb{D}^{1}$ and Jianhui Peng ${ }^{2}$ \\ ${ }^{1}$ School of Economics \& Management, Changsha University of Science \& Technology, Changsha 410114, China \\ ${ }^{2}$ School of Architecture, Harbin Institute of Technology, \\ Heilongjiang Cold Region Urban-Rural Human Settlements Science Key Laboratory, Harbin 150006, China
}

Correspondence should be addressed to Guojun Yin; 25786203@qq.com

Received 30 August 2021; Revised 28 September 2021; Accepted 30 September 2021; Published 14 October 2021

Academic Editor: Daqing Gong

Copyright (C) 2021 Guojun Yin and Jianhui Peng. This is an open access article distributed under the Creative Commons Attribution License, which permits unrestricted use, distribution, and reproduction in any medium, provided the original work is properly cited.

\begin{abstract}
The research on logistics heat facilitates the understanding of the drivers of regional logistics development. However, many scholars ignore the difference between prediction methods in terms of attributes and focal points of data analysis during the selection of regional logistics heat prediction model. Regional logistics interacts with regional economy. However, the studies on the coupling development between the two systems fail to make a detailed analysis in the light of their actual situation. Therefore, the evaluation of the coordination degree is often biased. To solve the problem, this paper probes into the prediction of regional logistics heat and the coupling development between regional logistics and economic systems. Firstly, an index system was established to measure the level of coupling development between the two systems, and a grey relational analysis was performed on the indices, leading to the evaluation results on coordination degree. Next, a composite model of GM $(1,1)$ and backpropagation (BP) neural network was proposed, and the deviation interval of the composite predictions was predicted based on Markov chain prediction model. The proposed algorithm proved effective through experiments.
\end{abstract}

\section{Introduction}

The logistics industry is an important support for the flow of economic elements and the junction between various parts of social production. It can promote both social development and economic benefits [1-3]. Logistics heat is the abstraction of specific geographical things, e.g., a logistics warehouse and a delivery point. The concept truthfully reflects the development of regional logistics and demonstrates the real-time logistics distribution with high credibility. Featured by timeliness, accuracy, scientific nature, and wide coverage, logistics heat should be studied to further understand the drivers of regional logistics development [4-6]. Regional logistics interacts with regional economy. Economic development expands the traffic network, improves the integration between transportation methods, and thus boosts the development of logistics industry [7-10].
Logistics heat has always been a hot topic in the research of the logistics industry [11-15]. Lan and Zhong [16] carried out entropy analysis and classification of the data collected from online logistics heat maps, aiming to optimize the spatial pattern of logistics in economic development zones. Wang [17] conducted logistic regression of the logistical network system, which centers on regional economic scale and logistics facilities, and obtained the description and analysis result on spatial logistics pattern, including service ability, service level, and radiation range. Based on logistics heat correlation index, Zhou and Zhang [18] analyzed the spatial pattern of logistics hubs at different levels and compared the internal spatial correlations within a region under different transportation conditions, market economy backgrounds, and policy support intensities. Lacoa et al. [19] summarized the development features of modern logistics packaging and e-commerce logistics against the rapid development of the big data and artificial intelligence (AI) and 
regarded green logistics and intelligent logistics as the direction of logistics transformation and upgrading. Ishii et al. [20] surveyed the logistics development state of Yangtze River Economic Belt and depicted the logistics heat distribution features at macro and micro levels. Wang et al. [21] modeled the coupling coordination degree between logistics and economy, identified the drivers of the logistics distribution features, and explored the spatiotemporal evolution law of logistics-economy coupling.

From the perspective of logistics heat, visualization tools can be adopted to analyze the spatiotemporal evolution pattern of regional economy and logistics heat at both macro and micro levels. This helps to discover the problems in regional logistics development and provide objective and authentic data support for relevant studies. Currently, most scholars in the industry refer to relevant research results to select the factors affecting the coupling development of regional logistics heat and economic systems, failing to analyze the specific conditions of the objects. That is why the coordination degree is often evaluated incorrectly [22]. Besides, many scholars ignore the difference between prediction methods in terms of attributes and focal points of data analysis, during the selection of regional logistics heat prediction model. To solve the problem, this paper introduces grey relational analysis and neural network into the prediction of regional logistics heat and the coupling development between regional logistics and economic systems. The main contents of this work are as follows: (1) setting up an index system to measure the level of coupling development between the two systems and carrying out grey relational analysis of all the indices; (2) evaluating the coordination degree of the coupling development between the two systems; (3) combining GM $(1,1)$ with backpropagation (BP) neural network into a hybrid prediction model for regional logistics heat; (4) estimating the deviation interval of the composite predictions, using the Markov chain prediction model. The proposed algorithm proved effective through experiments.

\section{Grey Relational Analysis and Coupling Development Evaluation}

2.1. Grey Relational Analysis. Grey relational analysis provides a quantitative metric for the development trend of a system. It is particularly suitable for analyzing dynamic processes. Before grey relational analysis, this paper draws on the existing research and selects the following evaluation indices, forming a systematic, scientific, operable, and stable index system to measure the level of coupling development between regional logistics system and regional economic system quantitatively and qualitatively:

(1) Mileage of regional transportation lines, $\mathrm{LE}_{1}$, which reflects the construction of regional logistics infrastructure.

(2) Fixed asset investment in logistics, $\mathrm{LE}_{2}$, which reflects the development potential of regional logistics industry.
(3) Number of logistics employees, $\mathrm{LE}_{3}$, which measures regional logistics development from the angle of manpower.

(4) Volume of freight traffic, $L_{4}$, which reflects the development scale of regional logistics.

(5) Total output of logistics industry, $\mathrm{LE}_{5}$, which reflects the contribution of logistics to regional economy.

(6) Growth rate of added value of logistics, $\mathrm{LE}_{6}$, which reflects the development speed and overall trend of regional logistics.

(7) Growth rate of logistics investment, $\mathrm{LE}_{7}$, which reflects the ability and trend of regional logistics attracting internal/external investment.

(8) Logistics development environment, $\mathrm{LE}_{8}$, which comprehensively reflects the development expectation of regional logistics.

(9) Logistics user satisfaction, $\mathrm{LE}_{9}$, which influences the subsequent development of regional logistics.

(10) Logistics talent cultivation, $\mathrm{LE}_{10}$, which reflects the training situation of professional talents.

(11) Regional gross domestic product (GDP), $\mathrm{LE}_{11}$, which reflects the overall level of regional economic development.

(12) Tertiary industry as a proportion of GDP, $\mathrm{LE}_{12}$, which reflects the advanced level of regional economic development.

(13) Total retail sales of consumer goods, $\mathrm{LE}_{13}$, which measures the changes in regional retail market and the prosperity of regional economy.

Indices 1-10 are about regional logistics development, and indices 11-13 are about regional economic development.

To prevent some indices from being ignored due to their units or dimensionality, the above index data should be normalized by

$$
\mathrm{LE}_{i}=\frac{\mathrm{LE}_{i}-\mathrm{LE}_{\text {min }}}{\mathrm{LE}_{\text {max }}-\mathrm{LE}_{\text {min }}}
$$

By (1), all the index data were converted into numbers in $[0,1]$. By taking the correlation coefficient between the contrastive series and the reference series as the maximum, the correlation coefficient between a regional logistics development index and a regional economic development index can be calculated by

$$
\delta_{i}(i)=\frac{\Delta \min +\delta_{\Delta \max }}{\Delta_{i}(i)+\delta_{\Delta \max }} .
$$

The grey correlation between indices equals the mean of the correlation coefficients obtained by (2):

$$
e_{i}=\frac{1}{M} \sum_{l=1}^{M} \delta_{i}(l) .
$$


2.2. Coupling Development Evaluation. With different attributes and relations, regional logistics system and regional economic system constitute a composite system of regional logistics-regional economy. The development states of the two systems determine the development coordination between them. Let $\mathrm{ZF}_{i}$ be the $i$-th system; let $\mathrm{YS}_{i}, \mathrm{ST}_{i}$, and $\mathrm{GN}_{i}$ be the environmental, structural, and functional elements of $\mathrm{ZF}_{i}$, respectively. The correlations, IR, between systems or within each system are diverse, interactive, hierarchical, and dynamic. These are natural multidimensional attributes within the systems. Let $h$ be the period measuring the time variation in each system. Then, whether the coordination degree between elements is reasonable can be characterized by

$$
\mathrm{ST}_{\mathrm{ZF}}=\left\{\mathrm{ZF}_{1}, \mathrm{ZF}_{2}, \mathrm{ZF}_{3} \cdots \mathrm{ZF}_{m}, \mathrm{IR}, h\right\} \text {, }
$$

where $m$ is the number of systems $(m \geq 2) ; \mathrm{ZF}_{i} \in\left\{\mathrm{YS}_{i}, \mathrm{ST}_{i}\right.$, $\left.\mathrm{GN}_{i}\right\}$.

Let $\mathrm{ZF}_{i}(h)$ be the sum of composite scores between systems in period $h ; \mathrm{ZF}_{m}(h-1)$ be the composite score of $\mathrm{ZF}_{i}$ in period $h-1$; and $\mathrm{YS}_{m}(h)$ be the composite effect of external environment on $\mathrm{ZF}_{i}$. Then, the relationship between systems can be measured by

$$
\begin{aligned}
\mathrm{ZF}_{i}(h)= & g_{i}\left[\mathrm{ZF}_{1}(h), \mathrm{ZF}_{2}(h), \mathrm{ZF}_{3}(h), \ldots, \mathrm{ZF}_{m}(h), \mathrm{ZF}_{m}(h-1), \mathrm{YS}_{m}(h)\right] \\
& (i=1,2, \ldots, m) .
\end{aligned}
$$

Let $\varepsilon_{i}(h)$ be the development state factor of each system in period $h ; \mathrm{ZF}(h)$ be the composite development state of the composite system in period $h$; and $\theta_{i}(h)$ be the weight of $\varepsilon_{i}(h)$. According to the definition of the coordination degree of the composite system, the coordinated development of the composite system can be quantified by

$$
\max \mathrm{ZF}(h)=\sum_{i=1}^{m} \theta_{i} \varepsilon_{i}(h) .
$$

Formula (6) shows that the better the development state and the higher the benefit of a system, the greater the value of $\varepsilon_{i}(h)$. Meanwhile, $\theta_{i}(h)$ characterizes the development state of that system on the composite system. On this basis, the order parameters of regional logistics system and regional economic system were configured. If the selected order parameters boost the benefits of the composite system, then the composite system has a positive effect; otherwise, the composite system has a negative effect.

Let $\operatorname{PA}\left(V_{i j}\right)$ be the effective contribution of the order parameter $A_{i j}$ to the corresponding system, and let $\mathrm{UL}_{i j}$ and $\mathrm{LL}_{i j}$ be the upper and lower bounds of the critical point of the order parameter $A_{i j}$ at the stable state of the composite system, respectively; i.e., $\mathrm{LL}_{i j} \leq A_{i j} \leq \mathrm{UL}_{i j}$. Then, the contribution of an order parameter to the development of the composite system can be described by

$$
\mathrm{PA}\left(V_{i j}\right)=\left\{\begin{array}{l}
\frac{A_{i j}-\mathrm{UL}_{i j}}{\mathrm{LL}_{i j}-\mathrm{UL}_{i j}} i=[1, k], \\
\frac{\mathrm{LL}_{i j}-A_{i j}}{\mathrm{LL}_{i j}-\mathrm{UL}_{i j}} i=[1, m] .
\end{array}\right.
$$

\section{Construction of Prediction Model}

3.1. GM $(1,1)$ Model. Figure 1 shows the cargo throughput and its growth rate at regional logistics centers. The scientific prediction of regional logistics heat is premised on the index system of the coupling development level between regional logistics system and regional economic system. Therefore, it is particularly important to select a suitable prediction method and understand the relevant issues of regional logistics heat. Based on correlation space and smooth discrete function, grey system theory defines grey derivative and grey differential equation and further establishes a dynamic model in the form of differential equation based on discrete data series. Based on the grey relational analysis results in the previous section, and the small sample size and nonlinearity of regional logistics heat, this paper combines GM $(1,1)$ with $\mathrm{BP}$ neural network into a composite prediction model to forecast the regional logistics heat, in the light of the coupling development between regional logistics system and regional economic system.

The applicability of GM $(1,1)$ model can be verified by the ratio test on the known series. The ratio of the initial values of index data $a^{(0)}=\left(a^{(0)}(1), a^{(0)}(2), \ldots, a^{(0)}(m)\right)$ can be calculated by

$$
\mu_{l}=\frac{a^{(0)}(l-1)}{a^{(0)}(l)}, \quad l=2,3, \ldots, m .
$$

If the index data meet the interval

$$
A=\left(e^{-2 / m+1}, e^{2 / m+1}\right),
$$

series $a^{(0)}$ can be calculated by setting up a $\operatorname{GM}(1,1)$ prediction model. Otherwise, the index data need to be converted by the rule

$$
\begin{aligned}
b^{(0)}(l)= & a^{(0)}(l)+d, \\
& l=1,2, \ldots, m,
\end{aligned}
$$

through accumulative generating operation (AGO) on the original series $a^{(0)}=\left(a^{(0)}(1), a^{(0)}(2), \ldots, a^{(0)}(m)\right)$ :

$$
\begin{aligned}
a^{(1)}(h)= & \sum_{l=1}^{h} a^{(0)}(l), \\
& l=1,2, \ldots, m .
\end{aligned}
$$




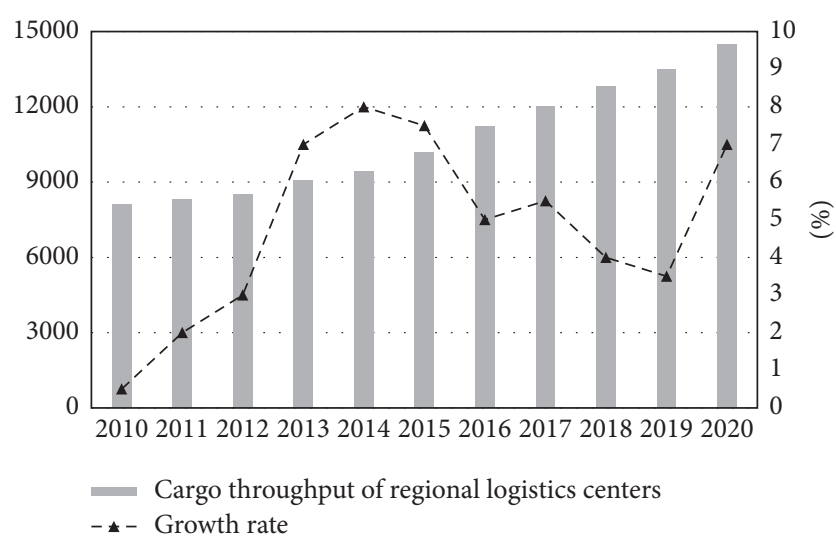

FIGURE 1: Cargo throughput and its growth rate at regional logistics centers.

Let DQ be the development coefficient that measures the trends of target series $a^{\prime(0)}$ and $a^{\prime(1)}$, and let $v$ be the grey action of the variation in index data. Then, the first-order linear differential equation can be constructed based on $a^{(1)}$ :

$$
\frac{\mathrm{d} a^{(1)}}{\mathrm{d} h}+\mathrm{DQ} \cdot a^{(1)}=v .
$$

Solving the differential equation by least squares method $W^{*}=(\mathrm{DQ}, v)^{T}=\left(O^{T} O\right)^{-1} O^{T} B_{m}$, we can obtain the desired $\mathrm{DQ}$ and $v$ :

$$
a^{(1)}(h+1)=\left\{a^{(0)}(1)-\frac{v}{\mathrm{DQ}}\right\} e^{-\mathrm{DQ} \cdot t}+\frac{v}{\mathrm{DQ}} .
$$

By taking average of cumulative index data, the vector $O$ and constant term vector $B_{m}$ can be obtained as

$$
O=\left[\begin{array}{ccc}
-\frac{1}{2}\left[a^{(1)}(1)+a^{(1)}(2)\right] & \cdots & 1 \\
-\frac{1}{2}\left[a^{(1)}(2)+a^{(1)}(3)\right] & \cdots & 1 \\
\vdots & \ddots & 1 \\
-\frac{1}{2}\left[a^{(1)}(m-1)+a^{(1)}(m)\right] & \cdots & 1
\end{array}\right] B_{m}=\left[\begin{array}{c}
a^{(0)}(2) \\
a^{(0)}(3) \\
\vdots \\
a^{(0)}(m)
\end{array}\right] .
$$

Then, $a^{(1)}(h+l)$ can be solved by substituting DQ and $v$ into (11). To obtain the actual prediction $a^{{ }^{(0)}}(h+l)$ in period $h+1$, the data imported to the prediction model must go through the inverse AGO (IAGO), because the model has undergone AGO:

$$
a^{\prime(0)}(h+1)=a^{\prime(1)}(h+1)-a^{\prime(1)}(h) .
$$

Substituting the solution of $a^{(1)}(h+l)$ to $(15)$, the regional logistics heat can be predicted as

$$
a^{\prime(0)}(h+1)=\left(1-e^{\mathrm{DQ}}\right)\left[a^{(0)}(1)-\frac{v}{\mathrm{DQ}}\right] e^{-\mathrm{DQ} \cdot h} .
$$

The standard deviation $\mathrm{ZB}_{1}$ of the residual series can be calculated by

$$
\mathrm{ZB}_{1}=\sqrt{\frac{\sum_{l=1}^{m}\left(\sigma_{(l)}^{(0)}-\hat{\sigma}^{(0)}\right)^{1}}{m-1}} .
$$

The standard deviation $\mathrm{ZB}_{2}$ of the original index data series can be calculated by

$$
\mathrm{ZB}_{2}=\sqrt{\frac{\sum_{l=1}^{m}\left(a_{(l)}^{(0)}-\widehat{a}^{(0)}\right)^{2}}{m-1}},
$$

where

$$
\begin{aligned}
& \widehat{a}=\frac{1}{m} \sum_{l=1}^{m} a_{(l)}^{(0)}, \\
& \widehat{\sigma}=\frac{1}{m} \sum_{l=1}^{m} \sigma_{(l)} .
\end{aligned}
$$

The posterior error can be calculated by

$$
\mathrm{HE}=\frac{\mathrm{ZB}_{1}}{\mathrm{ZB}_{2}}
$$

The small error probability can be calculated by

$$
\mathrm{GV}=\mathrm{GV}\left\{\left|\sigma_{(l)}^{(0)}-\widehat{\sigma}^{(0)}\right|<0.67 S_{2}\right\},
$$

where

$$
\sigma_{(l)}^{(0)}=\left|a_{(l)}^{(0)}-a_{(l)}^{\prime(0)}\right|
$$

The accuracy of the prediction model can be divided into different levels according to indices $\mathrm{HE}$ and GV.

3.2. BP Neural Network. Figure 2 shows the structure of BP neural network. This paper sets up a BP neural network via the following steps.

Firstly, the connection weights of the neural network were randomly assigned in the interval of $(0,1)$. Then, the objective training error $\xi$, training accuracy $\varepsilon$, and maximum number of learning iterations $N$ were configured. Then, the input samples $\mathrm{LE}_{l}=\left(\mathrm{LE}_{1}^{l}, \mathrm{LE}_{2}^{l}, \ldots, \mathrm{LE}_{m}^{l}\right)$ and the expected output samples $O_{0}=\left(O_{1}^{l}, O_{2}^{l}, \ldots, O_{m}^{l}\right)$ were provided to the established neural network.

Let $\omega_{i j}$ be the connection weight between the input layer and the hidden layer and $\varphi_{j}$ be the output threshold of each hidden layer node. Then, the output $d_{j}$ of each hidden layer node can be calculated from the input $r_{j}$ of that node, using the activation function $\mathrm{g}$ :

$$
\begin{aligned}
& r_{j}=\sum_{i=1}^{m} \omega_{i j} \mathrm{LE}_{i}-\omega_{j}, \\
& d_{j}=g\left(r_{j}\right), \quad j=1,2, \ldots, t .
\end{aligned}
$$

Let $u_{j h}$ be the connection weight between the hidden layer and the output layer and $\eta_{h}$ be the output threshold of each output layer node. Then, the input $\mathrm{SR}_{h}$ of each output layer node can be given by 


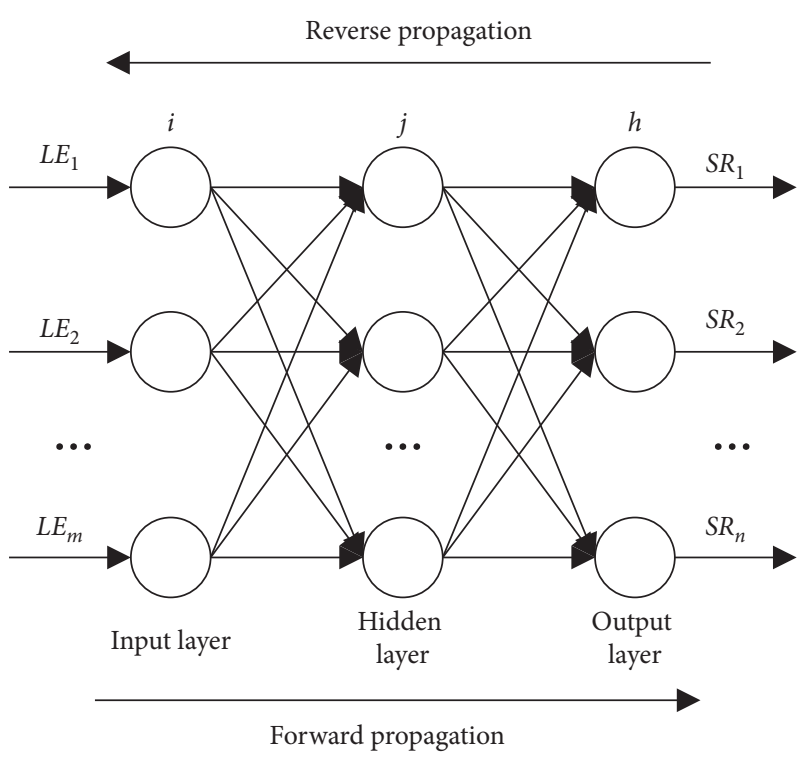

FIGURE 2: Structure of BP neural network.

$$
\mathrm{SR}_{h}=\sum_{j=1}^{m} u_{j h} d_{j}-\eta_{t}
$$

The output $\mathrm{SC}_{h}$ of each output layer node can be calculated by

$$
\begin{aligned}
\mathrm{SC}_{h}= & g\left(\mathrm{SR}_{h}\right), \\
& h=1,2, \ldots, w .
\end{aligned}
$$

The error function can be given by

$$
\zeta=\frac{1}{2} \sum_{h=1}^{w}\left(\xi_{h}-c_{h}\right)^{2} .
$$

Based on the calculated error, the partial derivative of the error function for output layer nodes could be obtained. Then, the neural network error will be backpropagated to the input layer. According to the error signal, each layer will update its weight. Thus, the errors of all layers could be iteratively adjusted. The iteration will stop when the error falls below the preset training accuracy, or the number of training iterations reaches the maximum number of learning iterations.

3.3. Composite Prediction Model. Considering the complexity of regional logistics system, it is very difficult to obtain historical development data over a long time. If GM $(1,1)$ and BP neural network could be combined, the composite prediction model would fit well with the features of regional logistics heat problem.

To improve the prediction effect of the composite model, it is important to assign a suitable weight to $\operatorname{GM}(1,1)$ and to BP neural network. Let $\theta_{i}$ be the weight of the i-th prediction method; $\zeta_{h}$ be the error of the composite series at time $h$; and $\zeta_{i(h)}$ be the error corresponding to the $i$-th prediction method at time $h$. Then, we have

$$
\zeta_{h}=K_{(h)}-a_{(h)}=\sum_{i=1}^{I} \theta_{i} \zeta_{i(h)} .
$$

To minimize the sum of squared errors (SSE) FH, a constrained objective function QFH can be established:

$$
\operatorname{MinFH}=\sum_{h=1}^{m}\left|\zeta_{h}^{2}\right|=\sum_{h=1}^{m}\left|\sum_{i=1}^{I} \theta_{i} \zeta_{i(h)}\right|,
$$

where $\theta_{i}$ satisfies the following equation:

$$
\sum_{i=1}^{I} \theta_{i}=1, \quad 0 \leq \theta_{i} \leq 1 .
$$

Then, the composite prediction model for regional logistics heat can be expressed as

$$
b_{h}=\theta_{1} K_{1}(h)+\theta_{2} K_{2}(h)+\ldots+\theta_{I} K_{I}(h) \text {. }
$$

3.4. Markov Chain Analysis. The Markov chain can predict the state of index data on regional logistics and economic systems at the next moment, according to the state and trend of the data, and mirror the fluctuations and instability of the index data. Taking the prediction error of regional logistics heat as a random variable, this paper estimates the deviation interval of the prediction by the GM $(1,1)-B P$ neural network composite model, using the Markov chain prediction model.

If the past is not correlated with a future random process $\left\{A_{i}(\theta), h \in \psi\right\}$, i.e., the future $\left(A\left(h_{m+1}\right)<a\right)$ is independent of the past $\left(A\left(h_{m+1}\right)=a_{m+1}, \ldots, A\left(h_{1}\right)=a_{1}\right)$; then, $\left\{A_{i}(\theta), h \in \psi\right\}$ has Markov property, and $\left\{A_{i}(\theta), h \in \psi\right\}$ can be called a Markov process. For any $h_{1}<h_{2}<\ldots<h_{m+1}, \quad h_{i} \in \psi$, $1 \leq i \leq m+1$, the conditional distribution of $A\left(h_{m+1}\right)$ relative to $A\left(h_{1}\right), A\left(h_{2}\right), \ldots, A\left(h_{m}\right)$ can be given by

$$
\begin{aligned}
\operatorname{GV}\left(A\left(h_{m+1}\right)\right. & \left.\leq a \mid A\left(h_{m}\right)=a_{m}, \ldots, A\left(h_{1}\right)=a_{1}\right) \\
& =\operatorname{GV}\left(A\left(h_{m+1}\right) \leq a \mid A\left(h_{m}\right)=a_{m}\right) .
\end{aligned}
$$

Time and state are discrete Markov processes, forming a Markov chain of random variables. The chain is stochastic and stationary, with no after-effect. Let $\mathrm{GV}_{c h}$ be the probability that the previous state transfers to the current state $h$ under condition $c$. Then, the transfer can be described by the transfer probability $\mathrm{GV}$ :

$$
\mathrm{GV}_{c h}=\mathrm{GV}\left\{a_{i}=h \mid a_{i-1}=c\right\} .
$$

The predicted regional logistic heat has multiple states, $\mathrm{ST}_{1}, \mathrm{ST}_{2}, \ldots, \mathrm{ST}_{m}$, at different moments. Therefore, many different situations may occur during the state transfer. The conditional probabilities under different situations constitute a transfer probability matrix. Let $\mathrm{GV}_{i j}$ be the state transfer probability from state $\mathrm{ST}_{i}$ to state $\mathrm{ST}_{j}$. Then, the transfer probability matrix $\mathrm{GV}$ can be described as 


$$
\mathrm{GV}=\left[\begin{array}{cccc}
\mathrm{GV}_{11} & \mathrm{GV}_{12} & \cdots & \mathrm{GV}_{1 m} \\
\mathrm{GV}_{12} & \mathrm{GV}_{22} & \cdots & \mathrm{GV}_{2 m} \\
\vdots & \vdots & \vdots & \vdots \\
\mathrm{GV}_{m 1} & \mathrm{GV}_{m 2} & \cdots & \mathrm{GV}_{m m}
\end{array}\right]
$$

The elements $\mathrm{GV}_{i j}$ in matrix $\mathrm{GV}$ need to satisfy

$$
\begin{aligned}
0 & \leq \mathrm{GV}_{i j} \leq 1, \\
\sum_{j=1}^{m} \mathrm{GV}_{i j} & =1, \quad(i=1,2, \ldots, m) .
\end{aligned}
$$

Since Markov chain has no after-effect, a multistep state transfer probability matrix can be obtained as

$$
\begin{aligned}
A^{(m+1)} & =\mathrm{GV} \cdot A^{(m)}, \\
A^{(m)} & =\mathrm{GV}^{(m)} A^{(0)} .
\end{aligned}
$$

\section{Experiments and Results Analysis}

The research data comes from the panel data on Yangtze River Economic Belt in 2000-2020. Table 1 presents the calculated results on the grey correlations between regional logistics and economy. The established GM $(1,1)$ prediction model was tested by fitting the historical index data on the coupling development between regional logistics and economic systems in 2000-2020. Based on the predicted regional logistics heat, the fitting effect is shown in Figure 3. The prediction error of $\mathrm{GM}(1,1)$ prediction model was 0.0984. The results of posterior error test were $\mathrm{ZB}_{1}=1957421, \quad \mathrm{ZB}_{2}=15144284$, posterior error was $\mathrm{HE}=0.145$, and small probability error was $\mathrm{GV}>0.9$. Therefore, the proposed GM $(1,1)$ prediction model has a high accuracy and, to a certain extent, reflects the future trend of logistics heat scale in the study area.

Figure 4 displays the change curve of training error of BP neural network. The training error curve started to converge at around the $300^{\text {th }}$ iteration. When the prediction accuracy of regional logistics heat reached $8.94 * 10^{-7}$, the preset prediction accuracy was achieved, and the iteration terminated. Table 2 presents the prediction results and errors of our composite prediction model, which makes full use of the predictions by GM $(1,1)$ and BP neural network. The two prediction models were integrated by least mean squares (LMS) method. In addition, L1 or L2 term was added to the loss function of the neural network, such that the network would try to minimize these terms. Through the additional L1 or L2 regularization, the network would limit the weight increment, because weight is a part of the loss function. Besides, the network became more generalizable, because it always tries to minimize the loss function. The weights of GM $(1,1)$ and BP neural network were set to 0.1945 and 0.8055 , respectively, aiming to minimize the MSE of the fitted prediction error.

To demonstrate its feasibility and effectiveness, our composite model was compared with $\operatorname{GM}(1,1)$ prediction model and BP neural network in terms of the prediction of
TABLE 1: Grey correlations between regional logistics and economy.

\begin{tabular}{lccc}
\hline Logistics indices & $\mathrm{LE}_{11}$ & $\mathrm{LE}_{12}$ & $\mathrm{LE}_{13}$ \\
\hline $\mathrm{LE}_{1}$ & 0.6452 & 0.2356 & 0.6827 \\
$\mathrm{LE}_{2}$ & 0.4589 & 0.1869 & 0.5403 \\
$\mathrm{LE}_{3}$ & 0.3932 & 0.3562 & 0.3426 \\
$\mathrm{LE}_{4}$ & 0.4241 & 0.1725 & 0.4075 \\
$\mathrm{LE}_{5}$ & 0.8318 & 0.2314 & 0.6421 \\
$\mathrm{LE}_{6}$ & 0.2876 & 0.3265 & 0.3028 \\
$\mathrm{LE}_{7}$ & 0.2163 & 0.3476 & 0.2212 \\
$\mathrm{LE}_{8}$ & 0.5231 & 0.1806 & 0.5063 \\
$\mathrm{LE}_{9}$ & 0.4962 & 0.1963 & 0.4256 \\
$\mathrm{LE}$ & 0.4035 & 0.2418 & 0.4136 \\
\hline
\end{tabular}

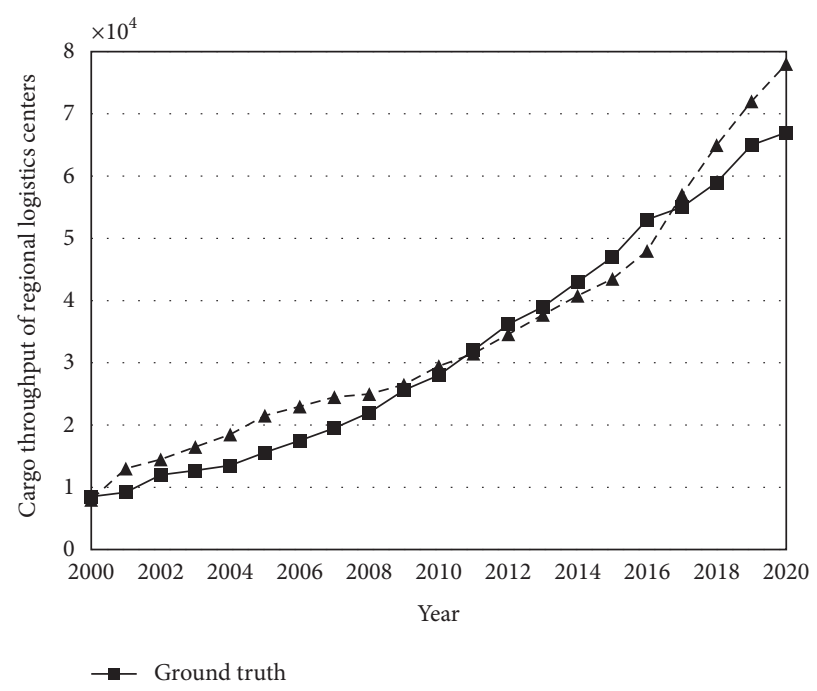

Figure 3: Training results of GM $(1,1)$ model.

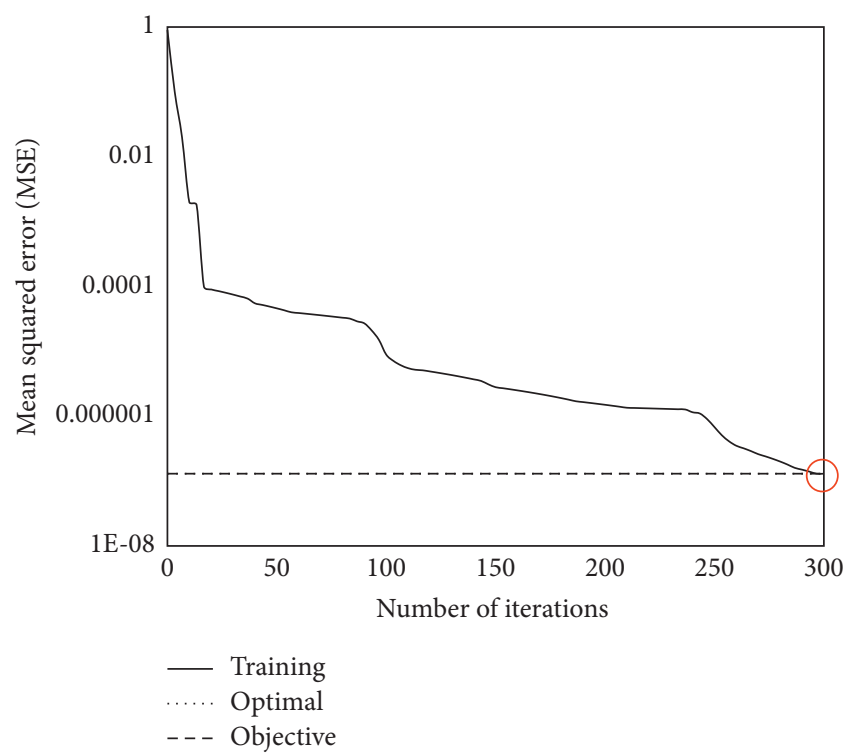

Figure 4: Change curve of training error of BP neural network.

regional logistics heat in 2010-2020. Table 3 compares the prediction results and errors of the three models. It can be seen that the composite model achieved the smallest error, the highest accuracy, and the most stable results. 
TABle 2: Prediction results and errors of our composite model.

\begin{tabular}{lccr}
\hline Year & Ground truth & Prediction & MSE \\
\hline 2015 & 49735 & 49871.6152 & 0.275 \\
2016 & 51647 & 52995.1563 & 0.261 \\
2017 & 51596 & 52351.9481 & 0.017 \\
2018 & 54273 & 54615.1506 & 0.006 \\
2019 & 61542 & 62362.4857 & 0.013 \\
2020 & 65131 & 65851.8416 & 0.011 \\
\hline
\end{tabular}

TABLE 3: Prediction results and errors of different models.

\begin{tabular}{|c|c|c|c|c|c|c|c|}
\hline \multirow{2}{*}{ Year } & \multirow{2}{*}{ Ground truth } & \multicolumn{2}{|c|}{ GM $(1,1)$} & \multicolumn{2}{|c|}{ BP neural network } & \multicolumn{2}{|c|}{ Composite model } \\
\hline & & Prediction & MSE & Prediction & MSE & Prediction & MSE \\
\hline 2010 & 8635 & 8672.25 & 0.431 & 8689.71 & 0.634 & 8676.64 & 0.482 \\
\hline 2011 & 10478 & 12603.72 & 20.287 & 9919.56 & -5.330 & 11632.12 & 11.015 \\
\hline 2012 & 12534 & 17521.83 & 39.794 & 10819.24 & -13.681 & 11941.36 & -4.728 \\
\hline 2013 & 14912 & 18654.31 & 25.096 & 11728.61 & -21.348 & 12896.65 & -13.515 \\
\hline 2014 & 16253 & 20156.74 & 24.019 & 14849.68 & -8.634 & 15794.72 & -2.820 \\
\hline 2015 & 18165 & 22513.95 & 23.941 & 16952.75 & -6.674 & 18984.24 & 4.510 \\
\hline 2016 & 22789 & 23891.56 & 4.838 & 24195.63 & 6.172 & 23673.36 & 3.881 \\
\hline 2017 & 26453 & 25234.79 & -4.605 & 28442.12 & 7.519 & 27984.21 & 5.788 \\
\hline 2018 & 31916 & 27919.32 & -12.522 & 29891.48 & -6.343 & 28561.25 & -10.511 \\
\hline 2019 & 32453 & 30917.08 & -4.733 & 34972.61 & 7.764 & 33849.64 & 4.304 \\
\hline 2020 & 36351 & 32156.26 & -11.540 & 37156.25 & 2.215 & 36894.76 & 1.496 \\
\hline
\end{tabular}

This paper treats the range of regional logistics heat as a random variable. The regional logistics heat is stochastic and stationary, with no after-effect. Based on the prediction errors of the three models above, the prediction results were divided by intervals of equal probability, such that the number of transfers between state intervals is reasonable, and the transfer rules are accurate. The state intervals of the specific prediction results were $\mathrm{ST}_{1}[4 \%, 8 \%], \mathrm{ST}_{2}[2 \%, 4 \%)$, $\mathrm{ST}_{3}[2 \%, 0.5 \%), \mathrm{ST}_{4}[-0.5 \%,-2 \%), \mathrm{ST}_{5}[-2 \%,-4 \%)$, and $\mathrm{ST}_{6}[-4 \%,-8 \%)$. Table 4 shows the prediction results on regional logistics heat and their errors and states.

Based on the states of the prediction results, the fitted results of the composite prediction model could be compiled into a one-step transfer Markov chain (Figure 5).

From the no-after-effect property of Markov chain, the multistep state transfer probability matrix can be obtained to reflect the variation in regional logistics heat. According to the states of prediction results in the years before the target years, the states of prediction results in the target years, number of transfer steps, and state transfer matrix could be derived by the prediction principle of Markov model. Table 5 shows the Markov chain analysis results of the composite prediction model.

As shown in Table 5, the state transfer probabilities in 2021-2023 mostly fell into the two intervals $\mathrm{ST}_{3}$ and $\mathrm{ST}_{4}$. In 2021, 2022, and 2023, the regional logistics heat was $4.577 * 10^{4}, 4.948 * 10^{4}$, and $5.689 * 10^{4}$, respectively. Through Markov chain analysis, the maximum probability intervals of the composite prediction model in the three years were $\left[5.015 * 10^{4}, 5.215 * 10^{4}\right], \quad\left[5.205 * 10^{4}\right.$, $\left.5.626 * 10^{4}\right]$, and $\left[5.694 * 10^{4}, 6.079 * 10^{4}\right]$, respectively. The ground truth of 2021 fell within $\left[5.015 * 10^{4}\right.$,
Table 4: Prediction results on regional logistics heat and their errors and states.

\begin{tabular}{lccc}
\hline & & \multicolumn{2}{c}{$\begin{array}{c}\text { Composite prediction } \\
\text { model }\end{array}$} \\
Year & Ground truth & -0.236 & $\mathrm{ST}_{5}$ \\
& & -7.205 & $\mathrm{ST}_{6}$ \\
2000 & 1536 & 1.895 & $\mathrm{ST}_{3}$ \\
2001 & 2109 & 7.823 & $\mathrm{ST}_{1}$ \\
2003 & 3512 & 2.215 & $\mathrm{ST}_{2}$ \\
2004 & 4173 & 3.546 & $\mathrm{ST}_{2}$ \\
2005 & 5236 & -3.592 & $\mathrm{ST}_{5}$ \\
2006 & 6014 & 4.194 & $\mathrm{ST}_{1}$ \\
2007 & 6531 & 3.682 & $\mathrm{ST}_{2}$ \\
2008 & 7983 & 2.918 & $\mathrm{ST}_{2}$ \\
2009 & 8324 & 1.812 & $\mathrm{ST}_{3}$ \\
2010 & 8542 & -7.613 & $\mathrm{ST}_{6}$ \\
2011 & 8635 & -4.782 & $\mathrm{ST}_{6}$ \\
2012 & 10478 & -3.319 & $\mathrm{ST}_{5}$ \\
2013 & 12534 & -3.842 & $\mathrm{ST}_{5}$ \\
2014 & 14912 & -1.389 & $\mathrm{ST}_{4}$ \\
2015 & 16253 & -0.914 & $\mathrm{ST}_{4}$ \\
2016 & 18165 & -0.237 & $\mathrm{ST}_{4}$ \\
2017 & 22789 & -1.589 & $\mathrm{ST}_{4}$ \\
2018 & 26453 & -1.791 & $\mathrm{ST}_{4}$ \\
2019 & 31916 & -2.985 & $\mathrm{ST}_{5}$ \\
2020 & 32453 & &
\end{tabular}

$5.215 * 10^{4}$ ], testifying the credibility of the predictions for 2022-2023.

According to the evolution trend of regional logistics demand predicted by our model, the cargo throughput in the study area will increase continuously. The outbreak of 


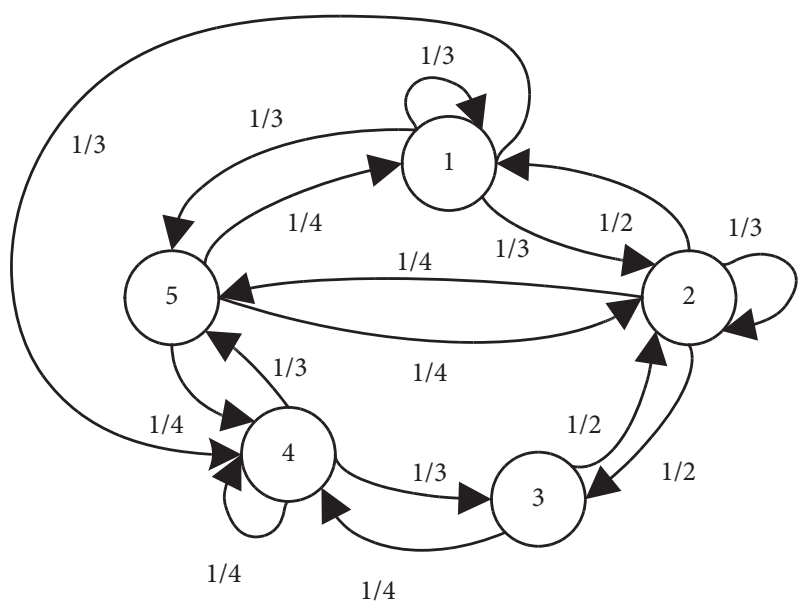

Figure 5: One-step transfer Markov chain.

TABLE 5: Markov chain analysis results of the composite prediction model.

\begin{tabular}{cccccc}
\hline Year & $\begin{array}{c}\text { Prediction } \\
\text { result }\end{array}$ & State & Prediction interval & Probability \\
\hline \multirow{4}{*}{2021} & & $\mathrm{ST}_{1}$ & $46,849.152$ & $48,416.052$ & $16.72 \%$ \\
& & $\mathrm{ST}_{2}$ & $48,496.545$ & $49,894.156$ & $12.34 \%$ \\
& & $\mathrm{ST}_{3}$ & $49,981.925$ & $50,893.525$ & $21.93 \%$ \\
& & $\mathrm{ST}_{4}$ & $50,156.513$ & $52,156.063$ & $21.02 \%$ \\
& $\mathrm{ST}_{5}$ & $52,950.258$ & $55,892.158$ & $11.64 \%$ \\
& & $\mathrm{ST}_{6}$ & $53,214.245$ & $56,412.245$ & $15.32 \%$ \\
\hline \multirow{4}{*}{2022} & & $\mathrm{ST}_{1}$ & $49,985.491$ & $52,819.564$ & $20.75 \%$ \\
& & $\mathrm{ST}_{2}$ & $52,895.987$ & $53,478.157$ & $12.68 \%$ \\
& & $\mathrm{ST}_{3}$ & $53,892.562$ & $54,314.156$ & $22.74 \%$ \\
& & $\mathrm{ST}_{4}$ & $52,059.364$ & $56,265.131$ & $20.38 \%$ \\
& & $\mathrm{ST}_{5}$ & $56,652.895$ & $59,491.564$ & $15.42 \%$ \\
& $\mathrm{ST}_{6}$ & $57,112.54$ & $31,245.23$ & $14.57 \%$ \\
\hline \multirow{4}{*}{2023} & $56,894.315$ & $\mathrm{ST}_{1}$ & $53,897.252$ & $56,792.256$ & $20.63 \%$ \\
& & $\mathrm{ST}_{2}$ & $56,394.956$ & $57,697.563$ & $13.12 \%$ \\
& & $\mathrm{ST}_{3}$ & $57,623.561$ & $58,318.395$ & $20.51 \%$ \\
& & $\mathrm{ST}_{4}$ & $56,948.213$ & $60,796.462$ & $21.16 \%$ \\
& $\mathrm{ST}_{5}$ & $60,156.332$ & $64,983.567$ & $16.38 \%$ \\
& & $\mathrm{ST}_{6}$ & $62,558.356$ & 67.412 & $9.28 \%$ \\
\hline
\end{tabular}

COVID-19 has caused the postponement or cancellation of most large-scale logistics activities and a drastic drop in foreign capital utilization in the short term. These negative effects will greatly impact the logistics service in the study area.

\section{Conclusions}

This paper introduces grey relational analysis and neural network into the prediction of regional logistics heat and the analysis of the coupling between regional logistics system and regional economic system. Specifically, an index system was constructed to measure the coupling development level between the two systems and to evaluate the grey correlations between the indices. Then, the coordination degree of the coupling development between the two systems was evaluated. Next, a composite prediction model for regional logistics heat was constructed by coupling GM $(1,1)$ with BP neural network. The Markov chain prediction model was employed to estimate the deviation intervals of the composite predictions. Through experiments, the change curve of the training results of $\operatorname{GM}(1,1)$ and the change curve of the training errors of BP neural network were plotted; the prediction results and errors were obtained for the proposed composite prediction model and compared with those of other prediction models. The comparison shows that the composite model achieved the smallest error, the highest accuracy, and the most stable results.

Due to the limitation of paper length and research ability, our research could be further expanded in many aspects. If conditions permit, the authors will split logistics heat into express delivery, logistics park, etc. and explore the factors affecting each component. The index system could also be enhanced and improved to cover more time profiles, yielding richer conclusions.

\section{Data Availability}

The data used to support the findings of this study are available from the corresponding author upon request.

\section{Conflicts of Interest}

The authors declare that they have no conflicts of interest.

\section{Acknowledgments}

This work was supported by the National-Social Science Fund Project "The Research on Mechanism and Countermeasures of Promoting Supply-Side Structural Reform through Logistics Institutional Mechanism Innovation" (Grant No. 17BJL084) and the Key Project of Hunan Education Department "The Research on Mechanism and Countermeasures of Promoting Supply-Side Structural Reform of Private Economy through Logistics Institutional Mechanism Innovation" (Grant No. 18Al18).

\section{References}

[1] T. H. Nazifa and K. K. Ramachandran, "Information sharing in supply chain management: a case study between the cooperative partners in manufacturing industry," Journal of System and Management Sciences, vol. 9, no. 1, pp. 19-47, 2019.

[2] A. L. Awaga, W. Xu, L. Liu, and Y. Zhang, "Evolutionary game of green manufacturing mode of enterprises under the influence of government reward and punishment," Advances in Production Engineering \& Management, vol. 15, no. 4, pp. 416-430, 2020.

[3] T. H. Nazifa, S. F. Mohaed, and A. B. Amin, "A brief discussion on supply chain management in construction industry," Journal of System and Management Sciences, vol. 9, no. 1, pp. 69-86, 2019.

[4] H.-Y. Li, W. Xu, Y. Cui, Z. Wang, M. Xiao, and Z.-X. Sun, "Preventive maintenance decision model of urban transportation system equipment based on multi-control units," IEEE Access, vol. 8, pp. 15851-15869, 2020.

[5] H. S. Lopes, R. S. Lima, and F. Leal, "Simulation project for logistics of Brazilian soybean exportation," International 
Journal of Simulation Modelling, vol. 19, no. 4, pp. 571-582, 2020.

[6] C. Kogler and P. Rauch, "Game-based workshops for the wood supply chain to facilitate knowledge transfer," International Journal of Simulation Modelling, vol. 19, no. 3, pp. 446-457, 2020.

[7] S. Hanif, D. Mu, S. Baig, and K. M. Alam, "A correlative analysis of modern logistics industry to developing economy using the VAR model: a case of Pakistan," Journal of Advanced Transportation, vol. 2020, Article ID 8861914, 10 pages, 2020.

[8] L. Gao and H. Dou, "Inventory management of railway logistics park based on artificial neural network," Journal Européen des Systèmes Automatisés, vol. 53, no. 5, pp. 715-723, 2020.

[9] J. Mackolil and B. Mahanthesh, "Logistic growth and SIR modelling of Coronavirus disease (COVID-19) outbreak in India: models based on real-time data," Mathematical Modelling of Engineering Problems, vol. 7, no. 3, pp. 345-350, 2020.

[10] N. T. Co, P. D. Lan, P. D. Quoc Huy, and M. S. Li, "Heatinduced degradation of fibrils: exponential vs logistic kinetics," The Journal of Chemical Physics, vol. 152, no. 11, Article ID 115101, 2020.

[11] S. Sokhansanj, S. Mani, S. Tagore, and A. F. Turhollow, "Techno-economic analysis of using corn stover to supply heat and power to a corn ethanol plant-part 1: cost of feedstock supply logistics," Biomass and Bioenergy, vol. 34, no. 1, pp. 75-81, 2010.

[12] L. M. Bramer, J. Rounds, C. D. Burleyson et al., "Evaluating penalized logistic regression models to predict Heat-Related Electric grid stress days," Applied Energy, vol. 205, pp. 1408-1418, 2017.

[13] X. Li and Z. Li, "A hybrid prediction model for e-commerce customer churn based on logistic regression and extreme gradient boosting algorithm," Ingénierie des Systèmes d'Information, vol. 24, no. 5, pp. 525-530, 2019.

[14] L. Maier, M. Hartmann, S. Tischer, and O. Deutschmann, "Interaction of heterogeneous and homogeneous kinetics with mass and heat transfer in catalytic reforming of logistic fuels," Combustion and Flame, vol. 158, no. 4, pp. 796-808, 2011.

[15] D. Herbadji, N. Derouiche, A. Belmeguenai, A. Herbadji, and S. Boumerdassi, "A tweakable image encryption algorithm using an improved logistic chaotic map," Traitement $d u$ Signal, vol. 36, no. 5, pp. 407-417, 2019.

[16] S. L. Lan and R. Y. Zhong, "Coordinated development between metropolitan economy and logistics for sustainability," Resources, Conservation and Recycling, vol. 128, pp. 345-354, 2018.

[17] L. Wang, "Research on the impact of e-commerce to logistics economy: an empirical analysis based on Zhengzhou airport logistics," International Journal of Security and Its Applications, vol. 9, no. 10, pp. 275-286, 2015.

[18] L. Zhou and Q. Zhang, "Mode and network layout of biomass resource recycling logistics in China under cyclic economy," International Journal of Applied Environmental Sciences, vol. 8, no. 6, pp. 751-762, 2013.

[19] U. Lacoa, G. Velarde, M. Kay, E. Blanco, and D. E. Saloni, "Design and development of logistics models for residential and commercial biomass pellets for heat and power generation in the US," BioResources, vol. 12, no. 1, pp. 1506-1531, 2017.

[20] K. Ishii, T. Furuichi, A. Fujiyama, and S. Watanabe, "Logistics cost analysis of rice straw pellets for feasible production capacity and spatial scale in heat utilization systems: a case study in Nanporo town, Hokkaido, Japan," Biomass and Bioenergy, vol. 94, pp. 155-166, 2016.

[21] Y. Wang, X. Zhu, T. Lu, and A. Jeeva, "Eco-efficient based logistics network design in hybrid manufacturing/remanufacturing system in low-carbon economy," Journal of Industrial Engineering and Management, vol. 2013, pp. 200-214, 2013.

[22] O. Pirogova, M. Makarevich, O. Ilina, and V. Ulanov, "Optimizing trading company capital structure on the basis of using bankruptcy logistic models under conditions of economy digitalization," IOP Conference Series: Materials Science and Engineering, vol. 497, no. 1, Article ID 12129, 2019. 